\title{
Abdominal imaging findings in adult patients with Fontan circulation
}

\author{
Tae-Hyung Kim ${ }^{1,2} \cdot$ Hyun Kyung Yang ${ }^{3} \cdot$ Hyun-Jung Jang ${ }^{3} \cdot$ Shi-Joon Yoo ${ }^{4} \cdot$ Korosh Khalili $^{3} \cdot$ Tae Kyoung Kim $^{3}$
}

Received: 13 December 2017 / Revised: 4 February 2018 / Accepted: 12 February 2018 / Published online: 5 April 2018

(C) The Author(s) 2018

\begin{abstract}
The Fontan procedures, designed to treat paediatric patients with functional single ventricles, have markedly improved the patient's survival into adulthood. The physiology of the Fontan circuit inevitably increases systemic venous pressure, which may lead to multi-system organ failure in the long-term follow-up. Fontan-associated liver disease (FALD) can progress to liver cirrhosis with signs of portal hypertension. Focal nodular hyperplasia-like nodules commonly develop in FALD. Imaging surveillance is often performed to monitor the progression of FALD and to detect hepatocellular carcinoma, which infrequently develops in FALD. Other abdominal abnormalities in post-Fontan patients include protein losing enteropathy and pheochromocytoma/paraganglioma. Given that these abdominal abnormalities are critical for patient management, it is important for radiologists to become familiar with the abdominal abnormalities that are common in post-Fontan patients on cross-sectional imaging.

\section{Teaching points}

- Fontan procedure for functional single ventricle has improved patient survival into adulthood.

- Radiologists should be familiar with unique imaging findings of Fontan-associated liver disease.

- Focal nodular hyperplasia-like nodules commonly develop in Fontan-associated liver disease.

- Hepatocellular carcinoma, protein-losing enteropathy, pheochromocytoma/paraganglioma may develop.
\end{abstract}

Keywords Liver cirrhosis $\cdot$ Focal nodular hyperplasia $\cdot$ Heart defects, congenital $\cdot$ Diagnostic imaging $\cdot$ Digestive system neoplasms

\section{Introduction}

The Fontan procedure was first introduced for patients with tricuspid atresia to allow systemic blood return to enter the pulmonary arteries without passage through a ventricle in

Electronic supplementary material The online version of this article (https://doi.org/10.1007/s13244-018-0609-2) contains supplementary material, which is available to authorized users.

Tae Kyoung Kim

taekyoung.kim@uhn.ca

1 Department of Radiology, Seoul National University Hospital, 101 Daehak-ro, Jongno-gu, Seoul 03080, South Korea

2 Department of Radiology, Seoul National University College of Medicine, 103 Daehak-ro, Jongnogu, Seoul 03080, South Korea

3 Department of Medical Imaging, Toronto General Hospital, 585 University Avenue, Toronto, ON M5G 2N2, Canada

4 Department of Diagnostic Imaging, Hospital for Sick Children, 555 University Avenue, Toronto, ON M5J2L4, Canada
1968 by Fontan and Baudet $[1,2]$. Subsequently, the procedure has been applied for most forms of functional single ventricles [3-6]. Surgical modifications of the procedure along with advances in postoperative management led to prolonged patient survival, frequently into adulthood [7-9].

The Fontan circuit allows the entire systemic venous return to enter the pulmonary vascular bed directly, bypassing the right ventricle. There are two ways to connect the systemic venous return to pulmonary arteries: either through the right atrium (namely atriopulmonary circulation) or by direct connection of superior vena cava (SVC) and inferior vena cava (IVC) to pulmonary arteries (namely cavopulmonary circulation). Cavopulmonary circulation is subdivided according to the type of IVC connection to the pulmonary artery either by intra-atrial lateral tunnel conduit or external cardiac conduit [10]. Due to the absence of a pumping ventricle, the transmission of pulmonary vascular resistance to the systemic venous circulation inevitably leads to chronically elevated systemic venous pressure. Thus, all organ systems of the body are exposed to chronic venous congestion, potentially leading to multi-system organ failure [11-13]. 
Fontan-associated liver disease (FALD) has been recognised as the disease process of liver structure and function resulting from the Fontan circulation, excluding other plausible causes such as viral hepatitis or alcohol toxicity. FALD is a broad term encompassing hepatic parenchymal change, hypervascular regenerative hepatic nodules and ultimately hepatic cirrhosis [14] . FALD is considered one of the major non-cardiac determinants of mortality in adult Fontan patients [15-18]. Other abdominal abnormalities found in adult patients with Fontan circulation include signs of portal hypertension such as ascites or varices, protein losing enteropathy (PLE) and pheochromocytoma/paraganglioma.

Although the imaging features of abdominal abnormalities after Fontan operation have been described in several publications mostly for the paediatric population [19-22], abdominal imaging findings in adult patients with Fontan circulation have not been well described in detail. In this review, we summarise the cross-sectional imaging findings of the hepatic and extrahepatic abnormalities in the abdomen in adult patients with Fontan circulation.

\section{Fontan-associated liver disease}

Among the gastrointestinal system, the liver is particularly vulnerable to the elevated systemic venous pressure in Fontan circulation compared to other organ systems for four principal reasons [13, 14]. Firstly, most venous return from the gastrointestinal system passes through the liver. Secondly, portal venous flow to the liver relies on the pressure gradient between the hepatic and portal veins that decreases as the systemic venous pressure increases, in contrast to other organs in which the blood flow relies on the pressure gradient between the arterial and venous systems. Third, the natural response of diversion of gastrointestinal blood to other organs such as brain, heart and muscles further compromises already precarious circulation of the liver. Lastly, lymphatic drainage disturbance due to increased central venous pressure causes lymphatic-mediated hepatic congestion and sinusoidal dilatation, which in turn attributes to the liver injury [23].

Adult patients with Fontan circulation are prone to developing FALD as they age. It frequently results in chronic hepatic congestion, liver cirrhosis, portal hypertension, focal nodular hyperplasia (FNH)-like nodules and even hepatocellular carcinoma (HCC) [15, 24-26]. The possible mechanisms of hepatic dysfunction in FALD include chronic passive venous congestion, reversed blood flow with deep intrahepatic reflux during atrial contraction [11], and reduced hepatic blood supply with subsequent hypoxic damage from relatively low cardiac output after Fontan operation [27, 28]. Histopathological changes begin with sinusoidal dilatation, parenchymal atrophy and progressive sinusoidal fibrosis in the perivenular distribution, similar to that of right heart failure, but more exaggerated over time in patients with Fontan circulation [11].

The severity of FALD is positively related to the duration of the Fontan circulation, elevated hepatic venous pressure, failing Fontan circulation and increased serological markers such as hyaluronic acid or gamma-glutamyltransferase [11, 29, 30]. Monitoring FALD with imaging surveillance is essential in guiding patient management such as medical therapy for liver cirrhosis, placement on the liver transplantation list or treating HCC when it is detected. In post-Fontan patients who are considered for heart transplantation, severe FALD is considered a contraindication for heart transplantation or combined heart-liver transplantation [16, 26, 31].

Given the clinical importance of FALD in adult patients with Fontan circulation, various non-invasive screening/ surveillance protocols for FALD have been suggested regarding the timing, intervals, and modalities [16, 18, 26, 32, 33]. Liver biopsy still remains the "gold standard" for assessing the severity of liver fibrosis and cirrhosis. However, reported liver changes secondary to FALD are not uniformly distributed and therefore liver biopsy may overestimate or underestimate the presence or nature of FALD [19, 28, 34]. Further, the risk of biopsy-related bleeding complication is likely to be higher due to the frequent use of warfarin and elevated systemic venous pressures [32, 35]. Therefore, non-invasive imaging surveillance may play a major role for the surveillance of FALD and its related changes.

\section{Imaging of hepatic parenchymal changes}

The liver may appear normal or slightly hypoechoic on ultrasound (US) at the early stage of congestive hepatopathy. As the fibrosis develops, coarse heterogeneous hyperechoic parenchymal pattern and surface nodularity are visualised [30] (Fig. 1a). The liver is often enlarged with caudate lobe hypertrophy similar to Budd-Chiari syndrome. Correlations have been reported between the severity of US features and the degree of hepatic fibrosis or cirrhosis [20, 36, 37]. Irregular parenchymal fatty infiltration with perivascular distribution can be seen in US and in magnetic resonance imaging (MRI) with in-phase and out-of-phase sequences (Fig. 1b-d) [22, 38]. On MRI, heterogeneous hyperintensity on T2-weighted images and hypointensity on T1-weighted images in the periphery of the liver, which is commonly seen in acute or subacute Budd-Chiari syndrome, is not seen in FALD [39-41], probably due to chronic gradual congestive changes of the liver over a long period. Diffusion-weighted imaging (DWI) can help estimating the degree of hepatic fibrosis. Wolff et al. [21] reported that low apparent diffusion coefficient values in DWI may reflect progressive liver damage due to chronic congestion and potential hypoperfusion in post-Fontan patients. 
Fig. 1 Hepatic parenchymal change in US in post-Fontan patients. a A 33-year-old woman with previous pulmonary atresia. US reveals heterogeneous parenchymal echotexture with central zone hyper-echogenicity and peripheral zone hypoyear-old woman with previous hypoplastic right ventricle syndrome. b US shows irregular like areas (arrowheads) surrounding portal vein branches with geographic margin. $\mathbf{c}, \mathbf{d}$ Inphase and opposed-phase axial T1-weighted MRI demonstrates increased signal intensity (SI) on in-phase image (c) and dropped SI on opposed-phase image (d), suggesting fat deposit (dotted to the hyperechoic mass-like areas with previous double outlet right ventricle. Contrast-enhanced ultrasound image obtained at 3 min shows marked coarse and heterogeneously weak delayed parenchymal enhancement echogenicity (arrows). b-d A 30homogeneous hyperechoic massarrows). These areas correspond on US. e A 48-year-old woman
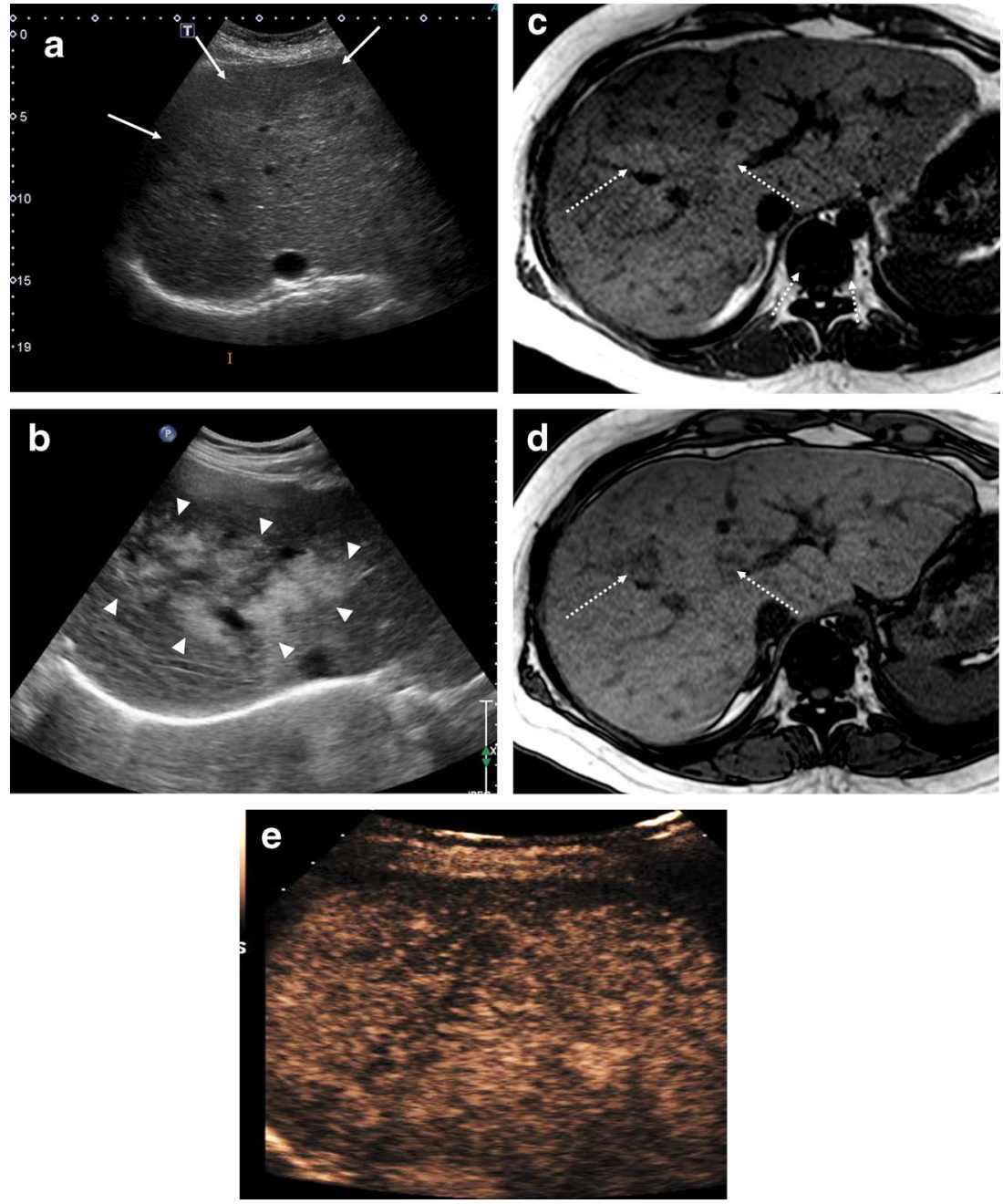

In addition to the morphological changes of the liver, markedly heterogeneous hepatic enhancement with mosaic or reticular patterns, resulting from relatively slow and poor enhancement near the congested hepatic veins, is the most common imaging feature in FALD [19, 38]. Contrastenhanced ultrasound (CEUS) in our anecdotal experience shows heterogeneous and decreased enhancement of the liver in the portal venous phase similar to cirrhotic liver of other common aetiologies (Fig. 1e). The abnormal enhancement is more prominent in the periphery of the liver than the central portion and is most evident in the portal venous phase (Fig. 2a-d). The hypertrophic caudate lobe often shows more homogeneous enhancement compared to the rest of the liver [13] (Fig. 2b). Hepatobiliary phase MRI using a hepatocytespecific contrast agent (gadoxetate disodium; Primovist; Bayer, Berlin, Germany) often shows heterogeneous hypoenhancement in the hepatobiliary phase, which reflects decreased hepatic function [42] (Fig. 2e). Hepatic fibrosis may be seen as reticular hyperenhancement in the delayed phase of CT or MRI. It has been reported that the degree of hepatic dysfunction is not well correlated with the degree of fibrosis in post-Fontan patients $[18,43]$. Therefore, follow-up with clinical and laboratory tests is also essential.

\section{Imaging of hepatic nodules}

Large regenerative hepatic nodules are commonly seen in adult patients with FALD. The pathophysiology of the development of the nodules is not fully elucidated, although it has been hypothesised that central venous hypertension transmitted directly to the hepatic peri-venular areas diminishes portal blood flow and promotes arterialisation [44-46]. The prevalence of the nodules in adult patients with Fontan circulation ranges from 20 to $30 \%$ [11, 44, 46]. The nodules have been named both large regenerative nodules and focal nodular hyperplasia (FNH)-like nodules in the literature and can be found in patients with other causes of hepatic venous outflow obstruction such as Budd-Chiari syndrome or right heart failure [38, 47-49]. The nomenclature of "FNH-like nodule" comes from imaging features [38, 46, 50] of hyperenhancement in the arterial phase and iso-enhancement in the delayed phase (Fig. 3a-c) and from histopathological 

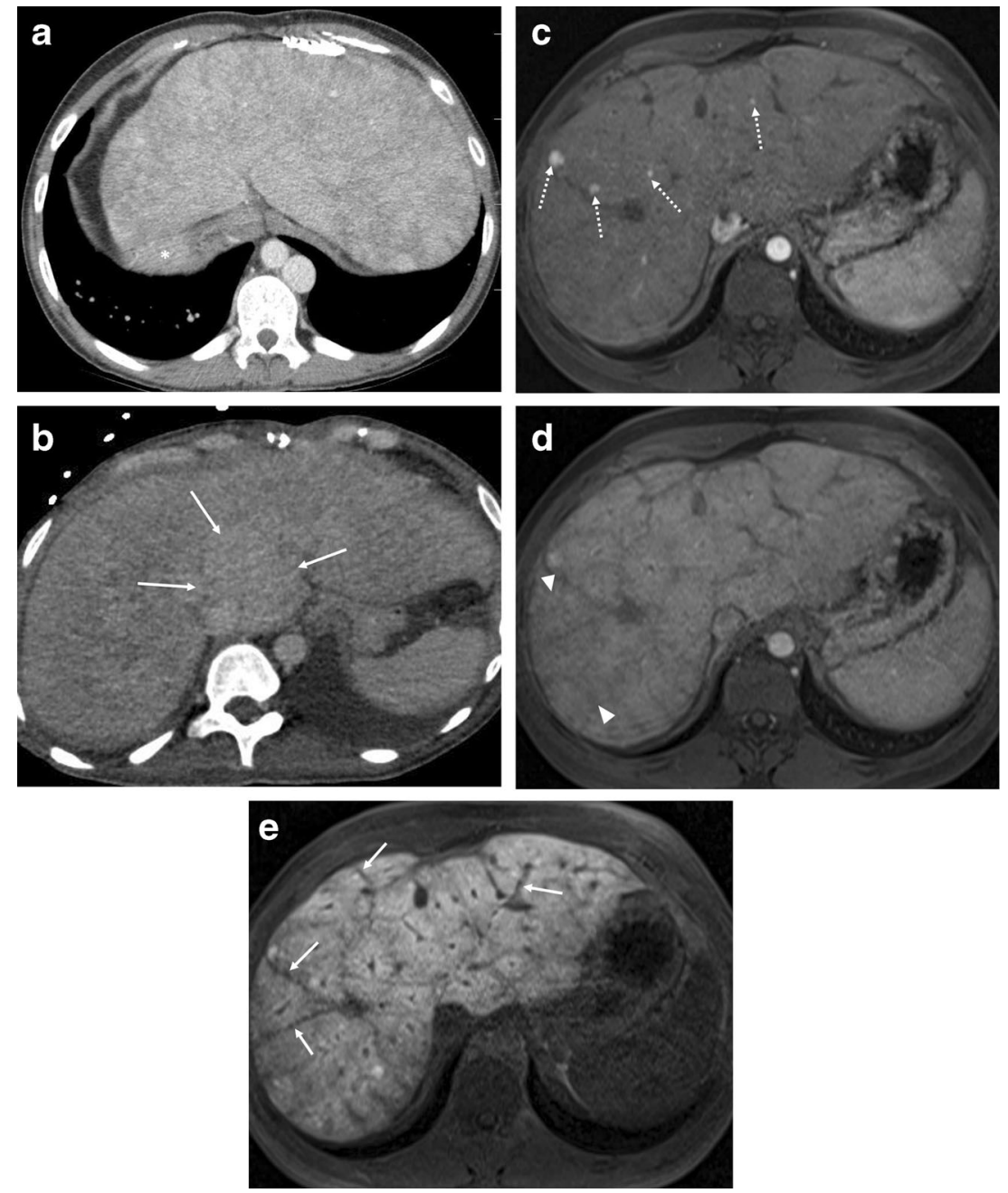

Fig. 2 Hepatic parenchymal change in CT and MRI in post-Fontan patients. a A 22-year-old woman with previous right atrial isomerism and pulmonary atresia. Axial CT image in portal venous phase demonstrates reticular regions of poor parenchymal enhancement in the periphery of the liver, which are prominent in the portal venous phase. Note that the stomach is located on the right side (asterisk) with left-sided liver and inferior vena cava (heterotoxy syndrome). b A 36-year-old woman with previous hypoplastic right ventricle syndrome. Axial CT image in portal venous phase demonstrates diffuse ill-defined inhomogeneous enhancement in the liver. Note that the caudate lobe hypertrophy with relative homogeneous attenuation (arrows). c-e A 22year-old woman with previous hypoplastic right ventricle syndrome. Axial contrast-enhanced T1-weighted MR images with gadoxetate

features [44] of small islands of hepatic parenchyma separated by bands of arterialised vascular fibrous septa with a pseudocapsule, similar to FNH.

Unfortunately, HCC can develop in adult patients with FALD [25, 26, 32, 46, 51] (Fig. 4) although the incidence of HCC is much lower than that of FNH-like nodules. Routine surveillance in FALD for HCC has been suggested, but there has been no consensus regarding the frequency of surveillance, the type of imaging modalities and the time interval after Fontan procedure. Progression from a dysplastic nodule to $\mathrm{HCC}$ is a potential stepwise pathway in liver cirrhosis disodium-enhanced MR images obtained in the arterial phase (c), transitional phase (d) and hepatobiliary phase (e). c Multiple small arterial-enhancing foci (dotted arrows) with contrast agent retention in the hepatobiliary phase are scattered in the liver, in keeping with focal nodular hyperplasia-like nodules. $\mathbf{d}$ The arterial-enhancing foci show no delayed washout. Note that heterogeneous parenchymal enhancement is prominent in the right posterior segment of the liver (arrowheads). e The liver shows heterogeneously mildly decreased contrast material uptake, possibly reflecting decreased hepatic function and congestion. Also, low-signal intensity reticular bands with no contrast material retention (arrows) which may be due to dilated veins / fibrous septa and tend to spare regions around the portal triads while coming into direct contact with hepatic veins

imposed by Fontan circulation. However, malignant potential of FNH-like nodules is uncertain and has been controversial $[18,24,44,46]$. Alleged benign large regenerative nodules in the chronic congested liver often mimic HCC radiologically and may render a correct diagnosis difficult [38]. Interval increase in size, washout in the portal venous phase, mosaic architecture, and elevated alpha-fetoprotein may be associated with HCC $[22,38]$.

FNH-like nodules in FALD are often small and multiple, and show a predilection for the right lobe in the periphery (mostly within $2 \mathrm{~cm}$ of the liver surface) [20]. On CEUS, 
Fig. 3 Focal nodular hyperplasialike nodules in post-Fontan patients. a-d A 21-year-old woman with previous double inlet left ventricle. a There is an approximately $2.3 \mathrm{~cm}$ homogeneous hypervascular nodule in segment 7 , periphery of the liver (arrow) on the axial arterial phase post contrast T1weighted MR image. b The lesion showed iso-signal intensity with no hypointensity on the axial transitional phase post contrast T1-weighted MR image. c The lesion with a few other small foci (dotted arrows) showing increased contrast material uptake in the hepatobiliary phase T1weighted MR image with gadoxetate disodium. d Singleshot echo-planar diffusionweighted image $(b=1,000 \mathrm{~s} /$ $\mathrm{mm}^{2}$ ) demonstrates no diffusion restriction in the corresponding lesion

Fig. 4 Hepatocellular carcinoma with extensive tumour thrombus in a post-Fontan patient. A 38year-old woman with previous right atrial isomerism and pulmonary atresia. a Axial CT image in the portal venous phase demonstrates a $1.1-\mathrm{cm}$ isoattenuated nodule causing surface contour abnormality in segment 2 of the liver (arrow). This lesion was initially reported as regenerative nodule. b-d On 6month follow-up MRI, the lesion increased to $3.4 \mathrm{~cm}$, showing arterial enhancement (b), washout in 3-min delay (c), and strong diffusion restriction in single-shot echo-planar diffusion-weighted image $\left(b=1,000 \mathrm{~s} / \mathrm{mm}^{2}\right)(\mathbf{d})$. Biopsy confirmed the lesion as $\mathrm{HCC}$ and the lesion was successfully treated with radiofrequency ablation (RFA). $\mathbf{e}$ Local recurrence of segment II in the dome HCC directly invaded the inferior vena cava (not shown) and subsequently an extensive tumour thrombus developed and filled the inferior vena cava, the right atrium (asterisks) and extended to the left main pulmonary artery
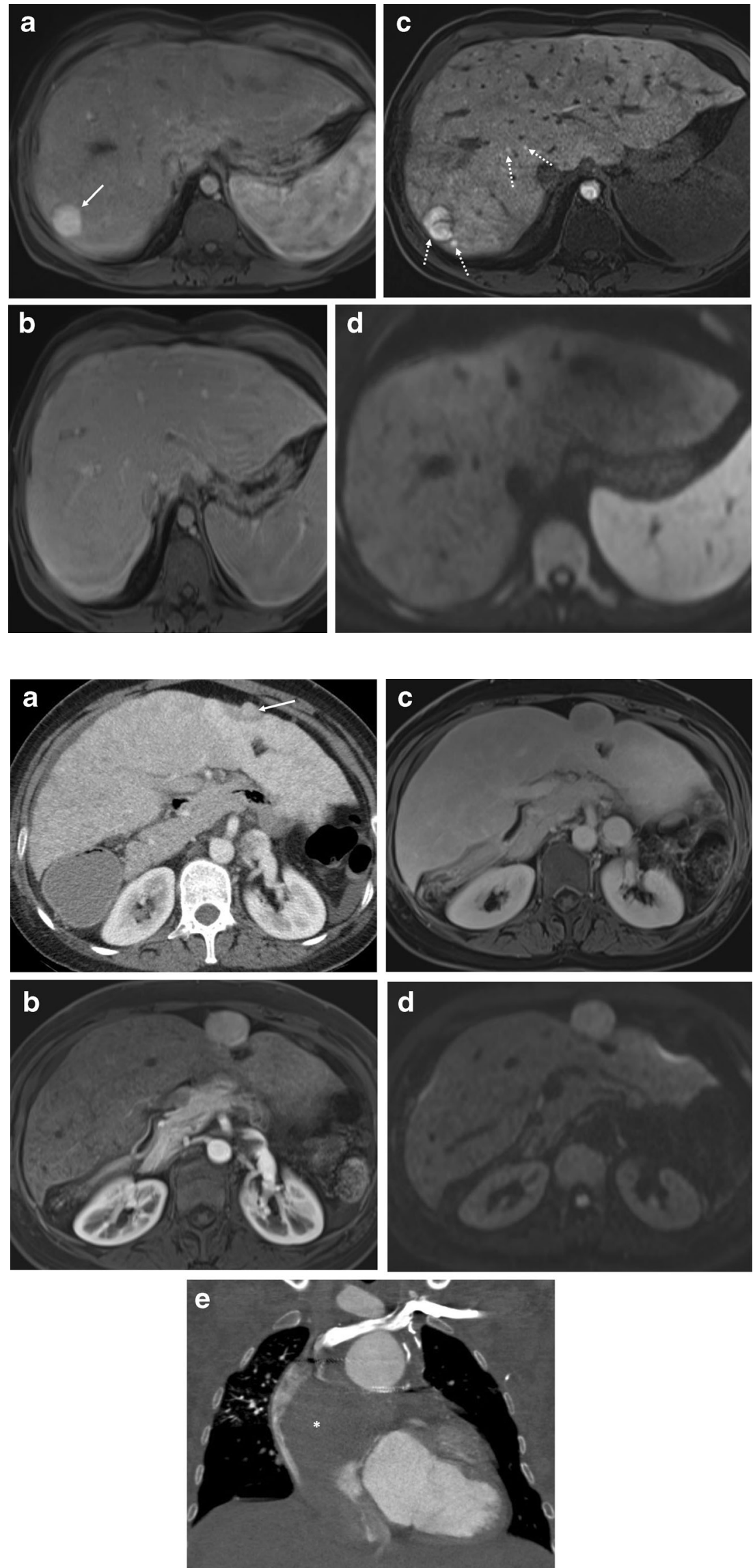
FNH-like nodules in FALD show enhancement features similar to FNH; hyper-enhancement in the arterial phase, centrifugal enhancement, central stellate vasculature and sustained enhancement without washout (Supplementary material 1). MRI using liver-specific contrast agent is useful for characterising FNH-like nodules by demonstrating iso- or hyper-enhancement in the hepatobiliary phase. The FNHlike nodules may show central hypointensity in the hepatobiliary phase representing a central scar (Fig. 3c).

HCC typically shows [25, 32, 51-57] arterial-phase hyperenhancement followed by late and mild washout on dynamic contrast-enhanced imaging. CEUS is potentially useful for indeterminate hepatic nodules in FALD on CT or MRI, especially for the patients who are contraindicated for contrastenhanced CT or MRI. Suspicion of HCC is raised upon large size, interval change in size or echogenicity, mass-like appearance or nodules causing contour abnormality on the liver surface (Fig. 4a). However, it is important to note that the FNH-like nodules can infrequently demonstrate washout in the delayed phase, mimicking HCC $[58,59]$ (Supplementary material 1). The reported incidence of delayed-phase washout in FNH-like nodules is approximately $10 \%$, in the setting of cardiac cirrhosis secondary to constrictive pericarditis and alcoholic cirrhosis. The pathophysiology for washout of FNH-like nodules is not clear. Wells et al. [22] speculated that the washout may not be related to an abnormality of the nodule itself, but a reflection of the background parenchymal contrast retention due to parenchymal congestion and fibrosis.

MRI using liver-specific contrast agent may be useful for the differentiation between FNH-like nodules and HCC, as HCC mostly show hypointensity in the hepatobiliary phase. DWI may be also useful in differentiating between HCC and FNH-like nodules. In our experience of a single case of $\mathrm{HCC}$ in FALD, HCC showed strong restricted diffusion, whereas FNH-like nodules did not show diffusion restriction, compatible with the study by Wells et al. [22] (Figs. 3d and 4d). The authors suggested other ancillary findings favouring benign FNH-like nodules, including hypointensity on T2-weighted images.

\section{Imaging of vascular abnormalities}

On Doppler US, normal hepatic vein shows a triphasic waveform, consisting of a hepatopetal (coming towards the liver) phase occurring during atrial systole and two hepatofugal phases related to atrial and ventricular diastole. Loss of the hepatopetal phase is typically considered to represent increased stiffness of the liver such as in hepatic fibrosis, steatohepatitis and cirrhosis [60-62].

Fontan operation inevitably alters hepatic venous waveform on Doppler US. The pattern of hepatopetal phase in hepatic vein differs between patients with total cavopulmonary anastomosis (including both lateral tunnels and extracardiac conduits) and with atriopulmonary connection [63-66] (Fig. 5a-e). Hepatopetal phase is preserved in atriopulmonary connection, which reflects the exclusion of atrial contribution to the venous circulation, whereas flow reversal is only visualised during early expiration in total cavopulmonary anastomosis. Also, similar to congestive heart failure, hepatic veins and IVC are dilated with abnormally increased pulsatility of the hepatic vein regardless of the anastomosis technique [38, 61, 67].

Portal venous waveform on Doppler US normally shows slightly phasic hepatopetal flow with mildly decreased flow during inspiration due to transient compression of the compliant liver parenchyma and easily collapsible portal venules and hepatic sinusoids $[61,68]$. In patients with Fontan circulation, the portal veins invariably show hepatopetal flow with variable phasicity, but the flow velocity is lower than in the normal population $[69,70]$. While the dilatation of the IVC and hepatic veins is frequently seen in post-Fontan patients, the diameter of the main portal vein and intrahepatic portal veins is usually small, possibly due to reduction of portal perfusion secondary to increased sinusoidal pressure with venous stasis [39] (Fig. 5). Bland portal vein thrombosis seems to be rare in post-Fontan patients with no reported cases compared to Budd-Chiari syndrome, where portal vein thrombosis occurs up to $18 \%[71$, 72]. Hepatofugal flow of the portal vein, which can be found in severe portal hypertension or Budd-Chiari syndrome with large extrahepatic portosystemic collaterals, has not been reported in post-Fontan patients $[63,66,70$, 73-75]. It can be explained by relatively high systemic venous pressure in Fontan circulation, preventing the development of large extrahepatic portosystemic collaterals.

On the arterial-phase CT with contrast injection through the upper extremity veins, dense opacification in the dependent portion of the IVC and hepatic veins is occasionally seen in post-Fontan patients. This is likely due to passive retrograde flow of the contrast material which is heavier than blood in the absence of effective right heart function (Supplementary material 2). This phenomenon might be different from the opacification of IVC and all hepatic veins in patients with right heart failure $[38,76,77]$, which is the result of reflux of contrast media into the inferior vena cava during right atrial contraction or tricuspid regurgitation.

\section{Extrahepatic findings}

\section{Signs of portal hypertension}

Extrahepatic complications of portal hypertension secondary to liver cirrhosis such as splenomegaly and ascites are commonly seen in adult Fontan patients. Compared to liver 
cirrhosis secondary to hepatitis, extrahepatic portosystemic shunts are uncommon or small in size in FALD, even in a decompensated state, likely due to the high systemic venous pressure in Fontan circulation and relatively low pressure gradient between the portal and systemic veins to promote the formation of large portosystemic collaterals [38] (Supplementary material 3).

\section{Protein losing enteropathy}

Protein losing enteropathy (PLE) is characterised by enteric loss of proteins including albumin, immunoglobulins and clotting factors. Patients with PLE present with peripheral oedema, ascites, diarrhoea, weight loss and malabsorption. The reported incidence of PLE in Fontan patients ranges from 3 to $18 \%$, with a reported mortality of $50 \%$ within 5 years after initial diagnosis [78-80]. Elevated systemic venous pressure and mesenteric vasoconstriction, which is the natural reaction to decreased cardiac output, might be the cause of PLE. Additionally, lymphatic dilatation, stasis and leakage, and inflammatory reaction secondary to elevated systemic venous pressure, further contribute to the development of PLE [19, 80, 81].

Early clinical diagnosis of PLE is often difficult because the patients may have a long asymptomatic period [80-82]. As multiple intensive therapeutic approaches enable an improved survival rate of up to $88 \%$ at 5 years [83], early recognition of excessive enteric loss of protein is important. Suggestive imaging findings including ascites, diffuse bowel wall thickening, and severe mesenteric oedema along the root of the mesentery on CT or MRI, should prompt a clinical evaluation to rule out PLE [13].
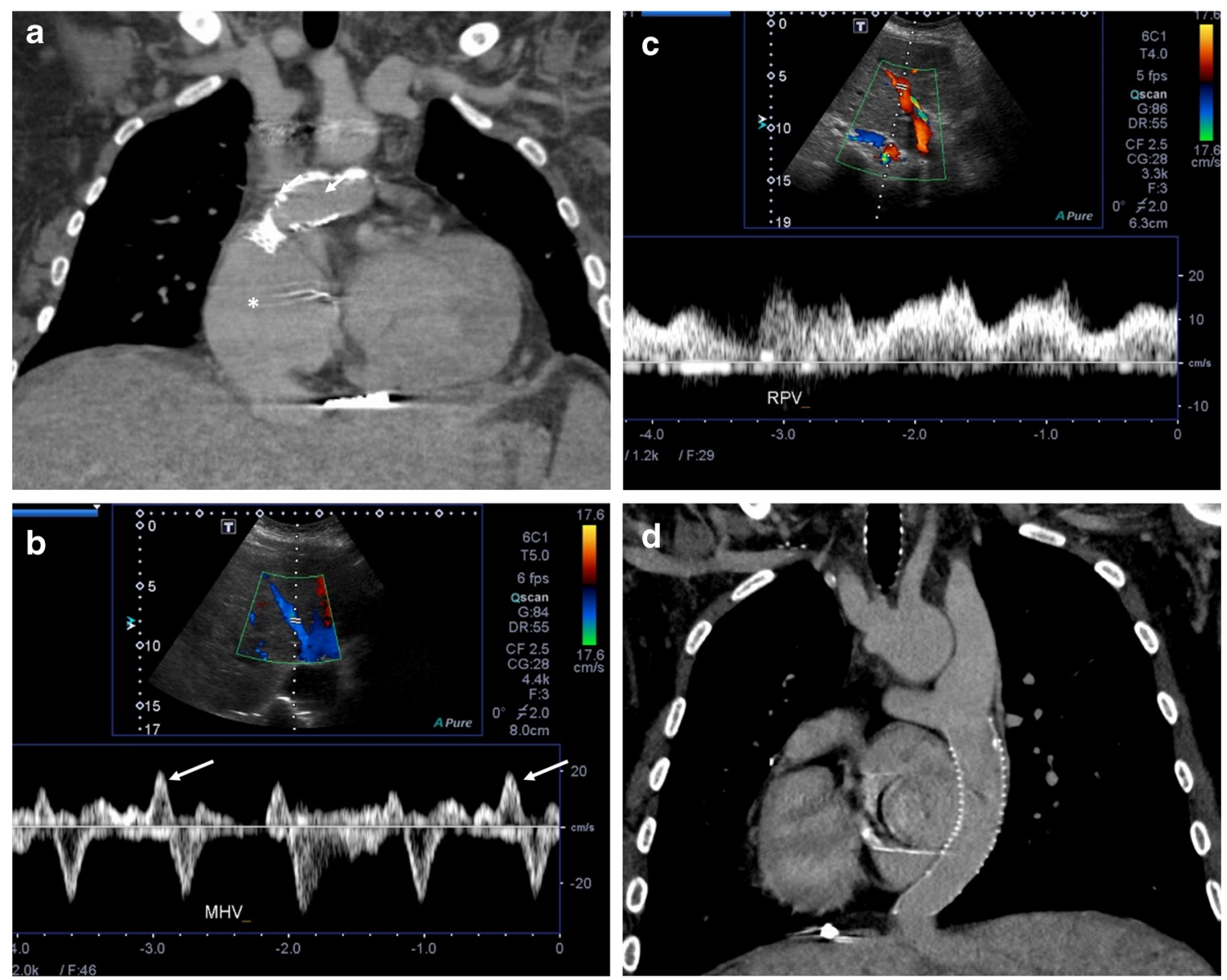

Fig. 5 Vessel evaluation in post-Fontan patients. a-c A 31-year-old woman with previous pulmonary atresia with intact ventricular septum. a Coronal CT shows indirect diversion of both SVC and IVC to pulmonary arteries via the right atrium (asterisk), corresponding with atriopulmonary anastomosis. b, c Spectral Doppler US examination. b Middle hepatic vein shows preservation of hepatopetal blood flow (arrows). c Right portal vein shows heterogeneously coarse hepatopetal flow. d-f A 21-year-old woman with previous left atrial isomerism with pulmonary atresia. $\mathbf{d}$ Coronal CT shows direct diversion of both the SVC and IVC to pulmonary arteries, corresponding with total cavopulmonary

anastomosis. Situs inversus is also noted. e, f Spectral Doppler US examination. e Left-side hepatic vein demonstrates disappearance of hepatopetal blood flow. f Right-side portal vein demonstrates relatively homogeneous hepatopetal flow. g-i A 33-year-old woman with previous hypoplastic right ventricle with tricuspid atresia. Coronal (g) and axial (h, i) CT images in the portal venous phases demonstrate intrahepatic venous-venous collateral formation (double-lined arrow in g), enlarged hepatic veins (arrowheads in $\mathbf{h}$ ), and small main portal vein (dotted arrow in i) 

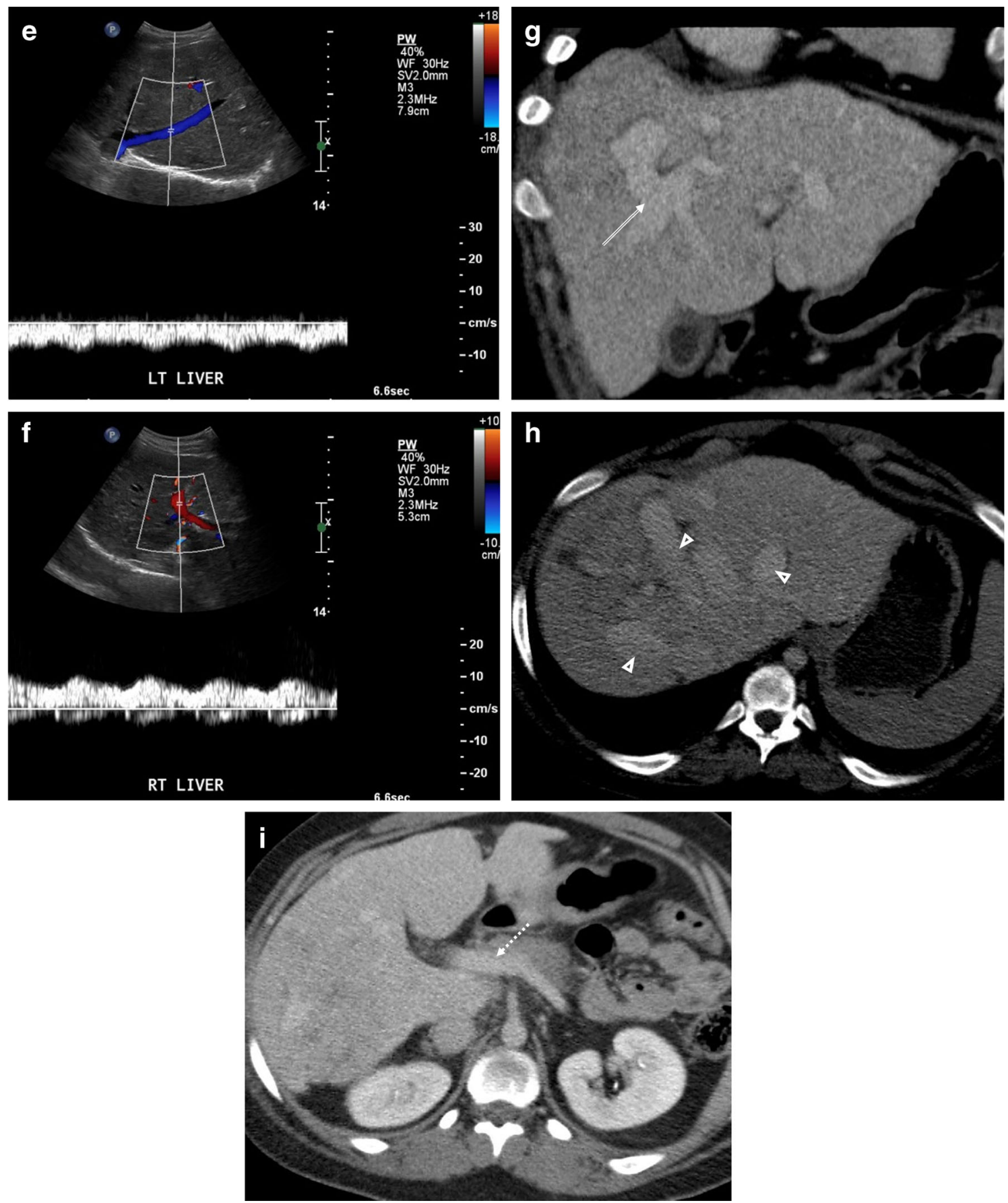

Fig. 5 (continued)

\section{Pheochromocytoma and paraganglioma}

Pheochromocytoma and paraganglioma are neuroendocrine tumours arising from neural crest-derived cells or organs in the adrenal gland and along the central sympathetic and parasympathetic chains, respectively. There have been several anecdotal reports of the development of pheochromocytoma or paraganglioma in patients with congenital cyanotic heart disease after corrective or palliative cardiac surgery, including the Fontan procedure [84-90]. Suggested pathophysiology is a stimulation of catecholamine overproduction and chronic endocrine hyperactivity resulting from the hypoxic state of congenital heart disease [91]. The tumours may cause palpitations, progressive arrhythmia, fatigue and malaise. Pheochromocytoma or paraganglioma are typically seen as heterogeneously and avidly hyper-enhancing masses with areas of necrosis and cystic component [92, 93] (Fig. 6). 

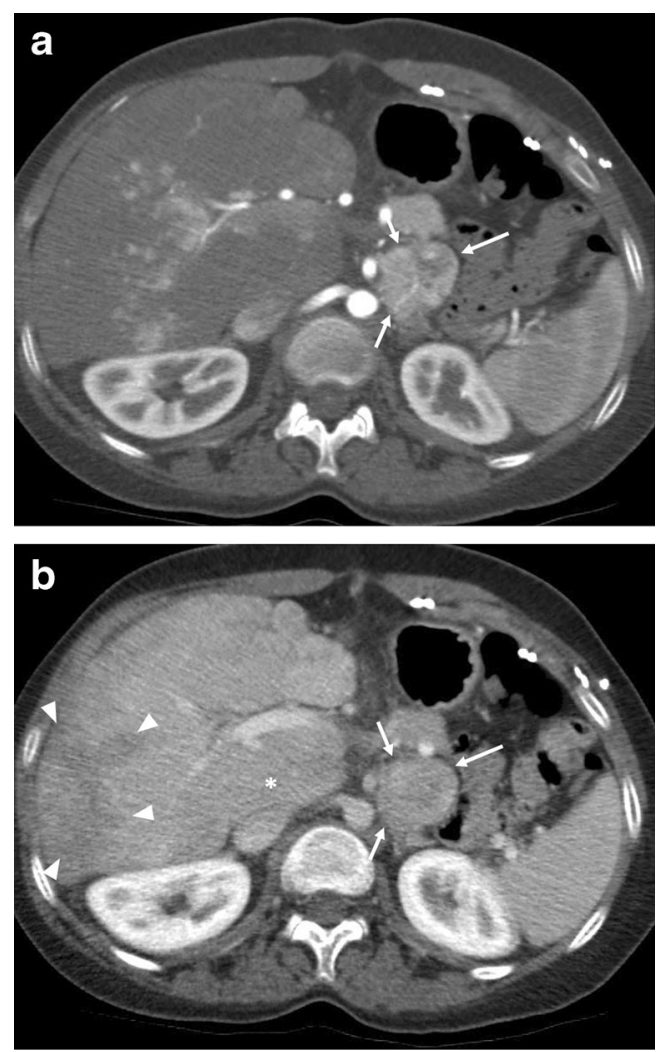

Fig. 6 Pheochromocytoma in a post-Fontan patient. A 24-year-old woman with previous hypoplastic right ventricle with dysplastic tricuspid valve. Axial CT images in the arterial (a) and portal venous (b) phases demonstrate a $4.5-\mathrm{cm}$ heterogeneous enhancing mass originating from left adrenal gland (arrows). The patient underwent an operation and the lesion was confirmed as pheochromocytoma. Note numerous arterial enhancing foci with no delayed washout scattered in the liver in the arterial phase and heterogeneous poor parenchymal enhancement in the periphery of the liver in the portal venous phase (arrowheads). Caudate lobe is hypertrophied with relative homogeneous enhancement (asterisk)

\section{Conclusions}

Fontan procedures, designed to treat patients with functional single ventricles, have markedly improved the patient's survival into adulthood. The physiology of the Fontan circuit inevitably increases systemic venous pressure, which may lead to multi-system organ failure in the long-term followup. FALD is most common among post-Fontan abdominal complications and can progress to liver cirrhosis with signs of portal hypertension. FNH-like nodules frequently develop in FALD as small multiple nodules with arterial-phase hyperenhancement and show imaging findings similar to FNH. Imaging surveillance is often performed to monitor the progression of FALD to liver cirrhosis and to detect HCC, which infrequently develops in FALD. Extrahepatic abdominal abnormalities in post-Fontan patients include signs of portal hypertension such as ascites, splenomegaly and varices, protein losing enteropathy, and pheochromocytoma/paraganglioma.
Given that these abdominal abnormalities are critical for patient management, it is important to be familiar with the typical imaging findings of the abnormalities on cross-sectional imaging.

Funding The authors state that this work has not received any funding.

\section{Compliance with ethical standards}

Guarantor The scientific guarantor of this publication is Dr. Tae Kyoung Kim.

Conflict of interest The authors of this manuscript declare no relationships with any companies whose products or services may be related to the subject matter of the article.

Statistics and biometry No statistical experience was required (review article).

Ethical approval Institutional Review Board approval was not required (review article).

\section{Methodology}

- Review article

- Performed at one institution

Open Access This article is distributed under the terms of the Creative Commons Attribution 4.0 International License (http:// creativecommons.org/licenses/by/4.0/), which permits unrestricted use, distribution, and reproduction in any medium, provided you give appropriate credit to the original author(s) and the source, provide a link to the Creative Commons license, and indicate if changes were made.

\section{References}

1. Fontan F, Mounicot FB, Baudet E, Simonneau J, Gordo J, Gouffrant JM (1971) "Correction" of tricuspid atresia. 2 cases "corrected" using a new surgical technic. Ann Chir Thorac Cardiovasc 10:39-47

2. Kreutzer G, Galindez E, Bono H, De Palma C, Laura JP (1973) An operation for the correction of tricuspid atresia. J Thorac Cardiovasc Surg 66:613-621

3. Humes RA, Feldt RH, Porter CJ, Julsrud PR, Puga FJ, Danielson GK (1988) The modified Fontan operation for asplenia and polysplenia syndromes. J Thorac Cardiovasc Surg 96:212-218

4. Mayer JE Jr, Helgason H, Jonas RA et al (1986) Extending the limits for modified Fontan procedures. J Thorac Cardiovasc Surg 92:1021-1028

5. Puga FJ (1991) Modified Fontan procedure for hypoplastic left heart syndrome after palliation with the Norwood operation. J Am Coll Cardiol 17:1150-1151

6. Russo P, Danielson GK, Puga FJ, McGoon DC, Humes R (1988) Modified Fontan procedure for biventricular hearts with complex forms of double-outlet right ventricle. Circulation 78:Iii20-Iii25

7. Anderson PA, Sleeper LA, Mahony L et al (2008) Contemporary outcomes after the Fontan procedure: a pediatric heart network multicenter study. J Am Coll Cardiol 52:85-98

8. Gersony WM (2008) Fontan operation after 3 decades: what we have learned. Circulation 117:13-15 
9. Pundi KN, Johnson JN, Dearani JA et al (2015) 40-year follow-up after the Fontan operation: long-term outcomes of 1,052 patients. J Am Coll Cardiol 66:1700-1710

10. Fredenburg TB, Johnson TR, Cohen MD (2011) The Fontan procedure: anatomy, complications, and manifestations of failure. Radiographics 31:453-463

11. Kiesewetter CH, Sheron N, Vettukattill JJ et al (2007) Hepatic changes in the failing Fontan circulation. Heart 93:579-584

12. Rychik J (2010) Forty years of the Fontan operation: a failed strategy. Semin Thorac Cardiovasc Surg Pediatr Card Surg Annu 13: 96-100

13. Yoo SJ, Prsa M, Schantz D et al (2014) MR assessment of abdominal circulation in Fontan physiology. Int J Cardiovasc Imaging 30: 1065-1072

14. Daniels CJ, Bradley EA, Landzberg MJ et al (2017) Fontanassociated liver disease: proceedings from the American College of Cardiology Stakeholders Meeting, October 1 to 2, 2015, Washington DC. J Am Coll Cardiol 70:3173-3194

15. Elder RW, McCabe NM, Hebson C et al (2013) Features of portal hypertension are associated with major adverse events in Fontan patients: the VAST study. Int J Cardiol 168:3764-3769

16. Lindsay I, Johnson J, Everitt MD, Hoffman J, Yetman AT (2015) Impact of liver disease after the fontan operation. Am J Cardiol 115: 249-252

17. Ohuchi H (2016) Adult patients with Fontan circulation: what we know and how to manage adults with Fontan circulation? J Cardiol 68:181-189

18. Rychik J, Veldtman G, Rand E et al (2012) The precarious state of the liver after a Fontan operation: summary of a multidisciplinary symposium. Pediatr Cardiol 33:1001-1012

19. Bulut OP, Romero R, Mahle WT et al (2013) Magnetic resonance imaging identifies unsuspected liver abnormalities in patients after the Fontan procedure. J Pediatr 163:201-206

20. Bae JM, Jeon TY, Kim JS et al (2016) Fontan-associated liver disease: spectrum of US findings. Eur J Radiol 85:850-856

21. Wolff D, van Melle JP, Dijkstra H et al (2016) The Fontan circulation and the liver: a magnetic resonance diffusion-weighted imaging study. Int J Cardiol 202:595-600

22. Wells ML, Hough DM, Fidler JL, Kamath PS, Poterucha JT, Venkatesh SK (2017) Benign nodules in post-Fontan livers can show imaging features considered diagnostic for hepatocellular carcinoma. Abdom Radiol (NY). https://doi.org/10.1007/s00261-017$1181-9$

23. Kendall TJ, Stedman B, Hacking N et al (2008) Hepatic fibrosis and cirrhosis in the Fontan circulation: a detailed morphological study. J Clin Pathol 61:504-508

24. Asrani SK, Asrani NS, Freese DK et al (2012) Congenital heart disease and the liver. Hepatology 56:1160-1169

25. Ghaferi AA, Hutchins GM (2005) Progression of liver pathology in patients undergoing the Fontan procedure: chronic passive congestion, cardiac cirrhosis, hepatic adenoma, and hepatocellular carcinoma. J Thorac Cardiovasc Surg 129:1348-1352

26. Nandwana SB, Olaiya B, Cox K, Sahu A, Mittal P (2018) Abdominal imaging surveillance in adult patients after Fontan procedure: risk of chronic liver disease and hepatocellular carcinoma. Curr Probl Diagn Radiol 47(1):19-22

27. Eipel C, Abshagen K, Vollmar B (2010) Regulation of hepatic blood flow: the hepatic arterial buffer response revisited. World $\mathrm{J}$ Gastroenterol 16:6046-6057

28. Pundi K, Pundi KN, Kamath PS et al (2016) Liver disease in patients after the Fontan operation. Am J Cardiol 117:456-460

29. Shimizu M, Miyamoto K, Nishihara Y et al (2016) Risk factors and serological markers of liver cirrhosis after Fontan procedure. Heart Vessel 31:1514-1521
30. Wu FM, Ukomadu C, Odze RD, Valente AM, Mayer JE Jr, Earing MG (2011) Liver disease in the patient with Fontan circulation. Congenit Heart Dis 6:190-201

31. Greenway SC, Crossland DS, Hudson M et al (2016) Fontanassociated liver disease: implications for heart transplantation. J Heart Lung Transplant 35:26-33

32. Josephus Jitta D, Wagenaar LJ, Mulder BJ, Guichelaar M, Bouman D, van Melle JP (2016) Three cases of hepatocellular carcinoma in Fontan patients: review of the literature and suggestions for hepatic screening. Int J Cardiol 206:21-26

33. Poterucha JT, Johnson JN, Qureshi MY et al (2015) Magnetic resonance Elastography: a novel technique for the detection of hepatic fibrosis and hepatocellular carcinoma after the Fontan operation. Mayo Clin Proc 90:882-894

34. Pariente D, Franchi-Abella S (2010) Paediatric chronic liver diseases: how to investigate and follow up? Role of imaging in the diagnosis of fibrosis. Pediatr Radiol 40:906-919

35. Manning DS, Afdhal NH (2008) Diagnosis and quantitation of fibrosis. Gastroenterology 134:1670-1681

36. Salvatore V, Borghi A, Peri E et al (2012) Relationship between hepatic haemodynamics assessed by Doppler ultrasound and liver stiffness. Dig Liver Dis 44:154-159

37. Zheng RQ, Wang QH, Lu MD et al (2003) Liver fibrosis in chronic viral hepatitis: an ultrasonographic study. World J Gastroenterol 9: 2484-2489

38. Wells ML, Fenstad ER, Poterucha JT et al (2016) Imaging findings of congestive Hepatopathy. Radiographics 36:1024-1037

39. Cura M, Haskal Z, Lopera J (2009) Diagnostic and interventional radiology for Budd-Chiari syndrome. Radiographics 29:669-681

40. Noone TC, Semelka RC, Siegelman ES et al (2000) Budd-Chiari syndrome: spectrum of appearances of acute, subacute, and chronic disease with magnetic resonance imaging. J Magn Reson Imaging 11:44-50

41. Soyer P, Rabenandrasana A, Barge J et al (1994) MRI of BuddChiari syndrome. Abdom Imaging 19:325-329

42. Yamada A, Hara T, Li F et al (2011) Quantitative evaluation of liver function with use of gadoxetate disodium-enhanced MR imaging. Radiology 260:727-733

43. Guha IN, Bokhandi S, Ahmad Z et al (2013) Structural and functional uncoupling of liver performance in the Fontan circulation. Int J Cardiol 164:77-81

44. Bryant T, Ahmad Z, Millward-Sadler H et al (2011) Arterialised hepatic nodules in the Fontan circulation: hepatico-cardiac interactions. Int J Cardiol 151:268-272

45. Shah H, Kuehl K, Sherker AH (2010) Liver disease after the Fontan procedure: what the hepatologist needs to know. J Clin Gastroenterol 44:428-431

46. Wallihan DB, Podberesky DJ (2013) Hepatic pathology after Fontan palliation: spectrum of imaging findings. Pediatr Radiol 43:330-338

47. Brancatelli G, Federle MP, Grazioli L, Golfieri R, Lencioni R (2002) Benign regenerative nodules in Budd-Chiari syndrome and other vascular disorders of the liver: radiologic-pathologic and clinical correlation. Radiographics 22:847-862

48. Brancatelli G, Vilgrain V, Federle MP et al (2007) Budd-Chiari syndrome: spectrum of imaging findings. AJR Am J Roentgenol 188:W168-W176

49. Ibarrola C, Castellano VM, Colina F (2004) Focal hyperplastic hepatocellular nodules in hepatic venous outflow obstruction: a clinicopathological study of four patients and 24 nodules. Histopathology 44:172-179

50. Brancatelli G, Federle MP, Grazioli L, Golfieri R, Lencioni R (2002) Large regenerative nodules in Budd-Chiari syndrome and other vascular disorders of the liver: CT and MR imaging findings with clinicopathologic correlation. AJR Am J Roentgenol 178: 877-883 
51. Asrani SK, Warnes CA, Kamath PS (2013) Hepatocellular carcinoma after the Fontan procedure. N Engl J Med 368:1756-1757

52. Jang HJ, Kim TK, Burns PN, Wilson SR (2015) CEUS: an essential component in a multimodality approach to small nodules in patients at high-risk for hepatocellular carcinoma. Eur J Radiol 84:1623-1635

53. Kim TK, Jang HJ (2014) Contrast-enhanced ultrasound in the diagnosis of nodules in liver cirrhosis. World J Gastroenterol 20: 3590-3596

54. Kim TK, Lee KH, Khalili K, Jang HJ (2011) Hepatocellular nodules in liver cirrhosis: contrast-enhanced ultrasound. Abdom Imaging 36:244-263

55. Rajoriya N, Clift P, Thorne S, Hirschfield GM, Ferguson JW (2014) A liver mass post-Fontan operation. QJM 107:571-572

56. Rosenbaum J, Vrazas J, Lane GK, Hardikar W (2012) Cardiac cirrhosis and hepatocellular carcinoma in a 13-year-old treated with doxorubicin microbead transarterial chemoembolization. J Paediatr Child Health 48:E140-E143

57. Saliba T, Dorkhom S, O'Reilly EM, Ludwig E, Gansukh B, AbouAlfa GK (2010) Hepatocellular carcinoma in two patients with cardiac cirrhosis. Eur J Gastroenterol Hepatol 22:889-891

58. Choi JY, Lee HC, Yim JH et al (2011) Focal nodular hyperplasia or focal nodular hyperplasia-like lesions of the liver: a special emphasis on diagnosis. J Gastroenterol Hepatol 26:1004-1009

59. Lee YH, Kim SH, Cho MY, Shim KY, Kim MS (2007) Focal nodular hyperplasia-like nodules in alcoholic liver cirrhosis: radiologicpathologic correlation. AJR Am J Roentgenol 188:W459-W463

60. Maruyama H, Yokosuka O (2017) Ultrasonography for noninvasive assessment of portal hypertension. Gut Liver 11:464-473

61. McNaughton DA, Abu-Yousef MM (2011) Doppler US of the liver made simple. Radiographics 31:161-188

62. Scheinfeld MH, Bilali A, Koenigsberg M (2009) Understanding the spectral Doppler waveform of the hepatic veins in health and disease. Radiographics 29:2081-2098

63. Hsia TY, Khambadkone S, Deanfield JE, Taylor JF, Migliavacca F, De Leval MR (2001) Subdiaphragmatic venous hemodynamics in the Fontan circulation. J Thorac Cardiovasc Surg 121:436-447

64. Hsia TY, Khambadkone S, Redington AN, De Leval MR (2000) Instantaneous pressure-flow velocity relations of systemic venous return in patients with univentricular circulation. Heart 83:583

65. Hsia TY, Khambadkone S, Redington AN, de Leval MR (2001) Effect of fenestration on the sub-diaphragmatic venous hemodynamics in the total-cavopulmonary connection. Eur J Cardiothorac Surg 19:785-792

66. Hsia TY, Khambadkone S, Redington AN, Migliavacca F, Deanfield JE, de Leval MR (2000) Effects of respiration and gravity on infradiaphragmatic venous flow in normal and Fontan patients. Circulation 102(19 Suppl 3):III148-III153

67. Moreno FL, Hagan AD, Holmen JR, Pryor TA, Strickland RD, Castle CH (1984) Evaluation of size and dynamics of the inferior vena cava as an index of right-sided cardiac function. Am J Cardiol 53:579-585

68. Moreno AH, Burchell AR (1982) Respiratory regulation of splanchnic and systemic venous return in normal subjects and in patients with hepatic cirrhosis. Surg Gynecol Obstet 154:257-267

69. Kutty SS, Peng Q, Danford DA et al (2014) Increased hepatic stiffness as consequence of high hepatic afterload in the Fontan circulation: a vascular Doppler and elastography study. Hepatology 59:251-260

70. Camposilvan S, Milanesi O, Stellin G, Pettenazzo A, Zancan L, D'Antiga L (2008) Liver and cardiac function in the long term after Fontan operation. Ann Thorac Surg 86:177-182

71. Bittencourt PL, Couto CA, Ribeiro DD (2009) Portal vein thrombosis and budd-Chiari syndrome. Clin Liver Dis 13:127-144

72. Darwish Murad S, Valla DC, de Groen PC et al (2006) Pathogenesis and treatment of Budd-Chiari syndrome combined with portal vein thrombosis. Am J Gastroenterol 101:83-90
73. Hsia TY, Khambadkone S, Bradley SM, de Leval MR (2007) Subdiaphragmatic venous hemodynamics in patients with biventricular and Fontan circulation after diaphragm plication. J Thorac Cardiovasc Surg 134:1397-1405 discussion 1405

74. Kaulitz R, Bergman P, Luhmer I, Paul T, Hausdorf G (1999) Instantaneous pressure-flow velocity relations of systemic venous return in patients with univentricular circulation. Heart 82:294-299

75. Schwartz MC, Glatz AC, Daniels K et al (2015) Hepatic abnormalities are present before and early after the Fontan operation. Ann Thorac Surg 100:2298-2304

76. Torabi M, Hosseinzadeh K, Federle MP (2008) CT of nonneoplastic hepatic vascular and perfusion disorders. Radiographics 28:1967-1982

77. Gore RM, Mathieu DG, White EM, Ghahremani GG, Panella JS, Rochester D (1994) Passive hepatic congestion: cross-sectional imaging features. AJR Am J Roentgenol 162:71-75

78. Feldt RH, Driscoll DJ, Offord KP et al (1996) Protein-losing enteropathy after the Fontan operation. J Thorac Cardiovasc Surg 112:672-680

79. Mertens L, Hagler DJ, Sauer U, Somerville J, Gewillig M (1998) Protein-losing enteropathy after the Fontan operation: an international multicenter study. PLE study group. J Thorac Cardiovasc Surg 115:1063-1073

80. Rychik J (2007) Protein-losing enteropathy after Fontan operation. Congenit Heart Dis 2:288-300

81. Meadows J, Jenkins K (2011) Protein-losing enteropathy: integrating a new disease paradigm into recommendations for prevention and treatment. Cardiol Young 21:363-377

82. Johnson JN, Driscoll DJ, O'Leary PW (2012) Protein-losing enteropathy and the Fontan operation. Nutr Clin Pract 27:375-384

83. John AS, Johnson JA, Khan M, Driscoll DJ, Warnes CA, Cetta F (2014) Clinical outcomes and improved survival in patients with protein-losing enteropathy after the Fontan operation. J Am Coll Cardiol 64:54-62

84. Bockelman HW, Arya S, Gilbert EF (1982) Cyanotic congenital heart disease with malignant paraganglioma. Cancer 50:2513-2517

85. Cheung YW, Spevack DM (2008) Single left ventricle and pheochromocytoma. Congenit Heart Dis 3:355-358

86. Hwang BH, Kim HY, Jung SE, Park KW (2012) Extra-adrenal pheochromocytoma after operation of congenital heart disease: a case report of 18-year-old boy. J Korean Surg Soc 83:65-68

87. Juneja R, Krishnamani NC, Kothari SS, Guleria S, Mahawar RM (2000) Pheochromocytoma and congenital cyanotic heart disease. Indian Heart J 52:452-454

88. Opotowsky AR, Moko LE, Ginns J et al (2015) Pheochromocytoma and paraganglioma in cyanotic congenital heart disease. J Clin Endocrinol Metab 100:1325-1334

89. Reynolds JL, Gilchrist TF (1966) Congenital heart disease and pheochromocytoma. Am J Dis Child 112:251-255

90. Sparks JW, Seefelder C, Shamberger RC, McGowan FX (2005) The perioperative management of a patient with complex single ventricle physiology and pheochromocytoma. Anesth Analg 100:972-975

91. Chung SJ, Lee YA, Shin CH, Yang SW, Bae EJ, Noh JI (2008) Pheochromocytoma associated with cyanotic congenital heart disease. Korean J Pediat 51:93-97

92. Blake MA, Kalra MK, Maher MM et al (2004) Pheochromocytoma: an imaging chameleon. Radiographics 24(Suppl 1):S87-S99

93. Lee KY, Oh YW, Noh HJ et al (2006) Extraadrenal paragangliomas of the body: imaging features. AJR Am J Roentgenol 187:492-504

\section{Publisher's Note}

Springer Nature remains neutral with regard to jurisdictional claims in published maps and institutional affiliations. 